\title{
THEORETICAL SIMULATION OF AIR CIRCULATION INSIDE CYCLONE MOUNTED AT EXHAUST OUTLET OF PNEUMATIC SEED DRILL TO OPTIMIZE IT
}

Gabriel Valentin Gheorghe, Marinela Mateescu, Catalin Persu, Iuliana Gageanu National Institute of Research-Development for Machines and Installations Designed to Agriculture and Food Industry, Romania gabrielvalentinghe@yahoo.com

\begin{abstract}
The present paper is based on CFD modelling of gas-solid flow in cyclone separators with different dust outlet geometries to analyse the flow characteristics and the cyclone performance. The cyclone performance parameters, collection efficiency, and pressure drop are obtained numerically. Changes in the particle trajectories due to the variation of the flow field inside the cyclone separators with the effect of the dust outlet section were also analysed in detail. Numerical study of the fluid flow and particle dynamics is presented by numerical techniques to characterize the performance of new cyclone separators. The design of this cyclone is based on the idea of improving the cyclone performance by increasing the vortex length. Cyclone separators are most often used in milling and are studied for these conditions. This article will study under the conditions imposed by a pneumatic seed drill to considerably reduce the removal of dust from the seeds into the atmosphere. The optimization of the cyclone separator will be done: by sizing the inlet and outlet pipes, by dimensioning the gauge of the cyclone or by obtaining the optimal air speed from the PTO of the tractor. This optimization will be made after studying these parameters analyzed with SolidWorks Flow Simulation. Computational fluid dynamics (either liquid or gas passing through or around an object) studies provide meaningful insight into the impact of the fluid flow, so you can address problems early, reduce the need for costly prototypes, and eliminate rework.
\end{abstract}

Keywords: theoretical simulation, seed drill, optimization.

\section{Introduction}

Toxic substances can be released into the atmosphere as a result of agricultural processes, especially those linked to fuel consumption during cultivation and to the use of pesticides [1-4]. Seed coating that controls pests by the application of small doses of pesticides directly on the seed is used on a large variety of crops [5]. Although this system is efficient and inexpensive, in recent years it has been restricted for some chemicals (e.g. neonicotinoids) because small amounts of dust abraded from the seed coating can be released into the atmosphere by sowing machines, which kills insects that are not harmful to crops including insects bringing important benefits including honey bees [6]. Because the seed coating is abraded inside the seeding element, all seed drills produce fine dust that could contaminate the environment. This is especially true for pneumatic drills, where an air stream generated by a fan is necessary to create a vacuum in the drill's sowing element. The air stream can blow solid dust particles detached from treated seeds towards areas adjacent to the field [7-12].

Various authors have studied this phenomenon and have measured dust emission or dust drift potential under controlled and repeatable conditions by different methods [13]. Based on those results, drills were classified according to drift risk [14].

In order to build a cyclone and to mount it on a seed drill, represented in Figure 1, an air and dust particles analysis was performed with SolidWorks Flow Simulation to observe some facts and to optimize the construction of the cyclone.

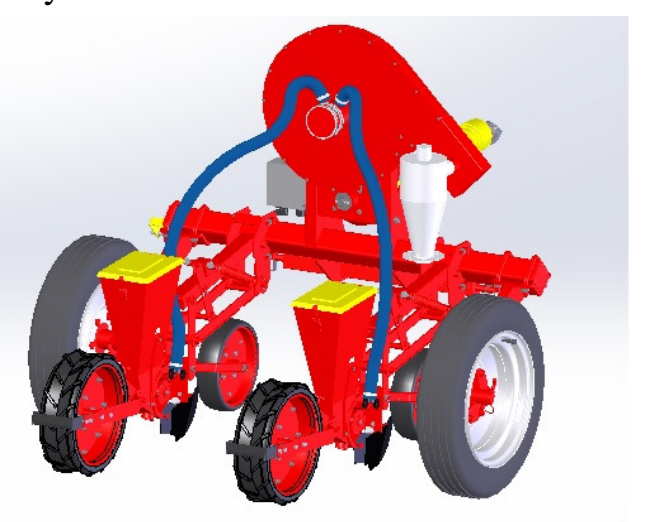

Fig. 1. Seed drill equipped with cyclone for dust collection 
There are different shaped cyclones, but the reversed flow cyclone, represented in Figure 2, is the most used model in industry, and from the point of view of the trace of the gas-dust mixture, the cyclones are inertial separators in curvilinear currents.

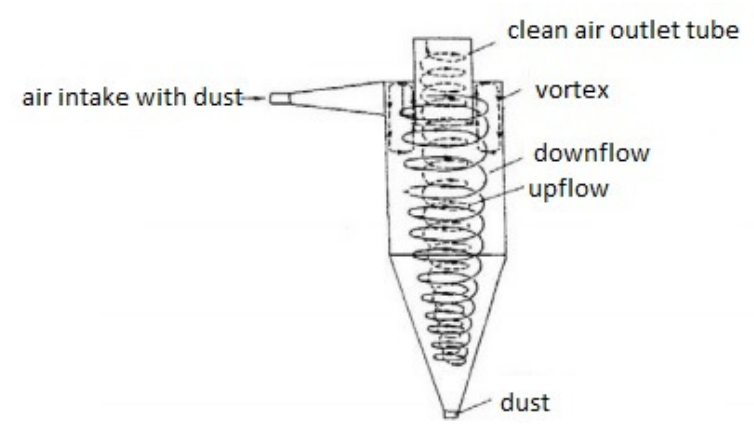

Fig. 2. Types of movements developed in cyclone

The link of the geometric shape of the cyclone is essential, with its efficiency given by the complexity of aerodynamic flow in these dust separators (Figure 2). In this sense the movement of the biphasic air-dust mixture into the cyclone is studied.

\section{Materials and methods}

Input data: the geometric dimensions of the cyclone (will do this simulation for different dimension of the cyclone to see, which geometric construction is the best); particle density of dust $\left(3200 \mathrm{~kg} \cdot \mathrm{m}^{-3}\right)$, particle diameter (between 1.5-50.0 $\mu \mathrm{m}$ ), average air inlet velocity in the cyclone and dynamic air viscosity.

At the entrance to the cyclone, the air has a temperature of $80^{\circ} \mathrm{C}$, velocity of $20 \mathrm{~m} \cdot \mathrm{s}^{-1}$ and dynamic air viscosity $21 \cdot 10^{-6} \mathrm{~kg} \cdot(\mathrm{m} \cdot \mathrm{s})^{-1}[15]$.

The first step is to introduce these data as simulation input parameters, as shown in Figure 3.

To continue, we need to calculate the mass flow (1), which can be calculated from the density of the substance, the cross-section through which the substance flows, and its velocity in the flow section.

$$
\dot{m}=\rho \cdot v \cdot A
$$

where $\dot{m}$ - mass flow rate (which will be introduced in input data of particle study alongside the particle diameter), $\mathrm{kg} \cdot \mathrm{s}^{-1}$;

$\rho$ - density of the substance $(3200), \mathrm{kg} \cdot \mathrm{m}^{-3}$;

$v$ - velocity in the flow section $(20), \mathrm{m} \cdot \mathrm{s}^{-1}$;

$A$ - cross-section through which the substance flows (0.001756), $\mathrm{m}^{2}$.

After the introduction of the air input data, we introduced the input data for the dust particles (Figure 4), along with the value that we calculated with the mass flow rate formula (1).

The simulation was run and the results were interpreted.

\begin{tabular}{|c|c|c|c|}
\hline General Settings & & & ? \\
\hline Parameter & Value & & \multirow{3}{*}{ Analysis type } \\
\hline Parameter Definition & User Defined & $\checkmark$ & \\
\hline$\boxminus$ Thermodynamic Parameters & & & \\
\hline Parameters & Temperature, density & $\checkmark$ & \multirow{2}{*}{$O_{2}$ Fluids } \\
\hline Temperature & $353 \mathrm{~K}$ & & \\
\hline Density & $1.295 \mathrm{~kg} / \mathrm{m}^{\wedge} 3$ & & \multirow{2}{*}{ Wall conditions } \\
\hline$\boxminus$ Velocity Parameters & & & \\
\hline ... Parameter & Velocity & $v$ & \multirow{4}{*}{ (1) Initial conditions } \\
\hline Velocity in $\mathrm{X}$ direction & $20 \mathrm{~m} / \mathrm{s}$ & & \\
\hline Velocity in $Y$ direction & $0 \mathrm{~m} / \mathrm{s}$ & & \\
\hline Velocity in $Z$ direction & $0 \mathrm{~m} / \mathrm{s}$ & & \\
\hline
\end{tabular}

Fig. 3. Input parameters of theoretical simulation

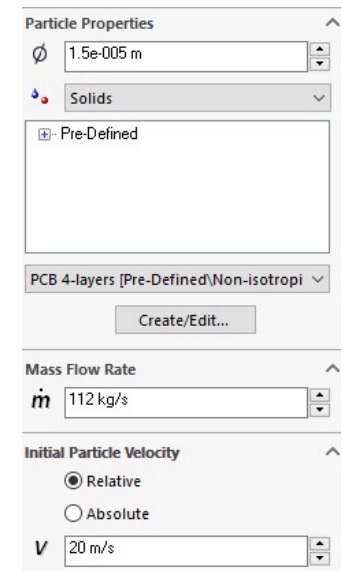

Fig. 4. Input data for dust particles 


\section{Results and discussion}

In the first image (Figure 5.a), one can observe the increase of the air velocity in the upper part from an input value of $20 \mathrm{~m} \cdot \mathrm{s}^{-1}$, some trajectories having a very large variation in the part, where the vortex is formed, so that at the exit it has the value of $5 \mathrm{~m} \cdot \mathrm{s}^{-1}$. In Figure 5.b, the movement of the dust is observed, which descends to the bottom of the cyclone and is captured in a bag and therefore in the upper part of the image we do not have particles of dust.

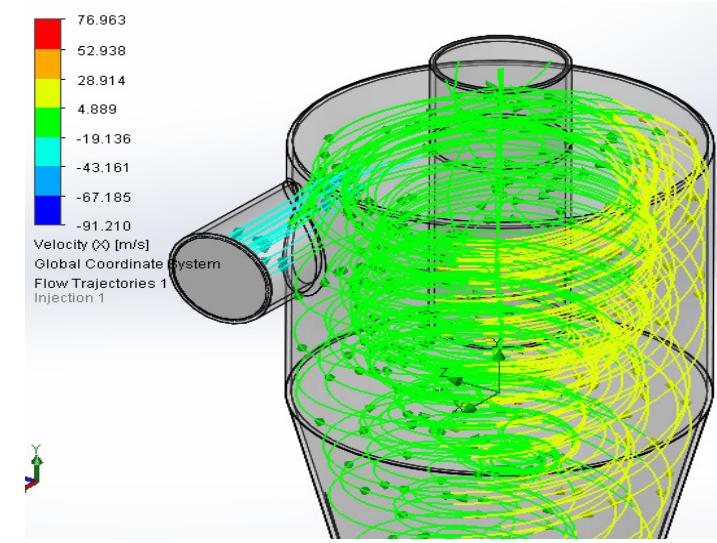

a) Air trajectory

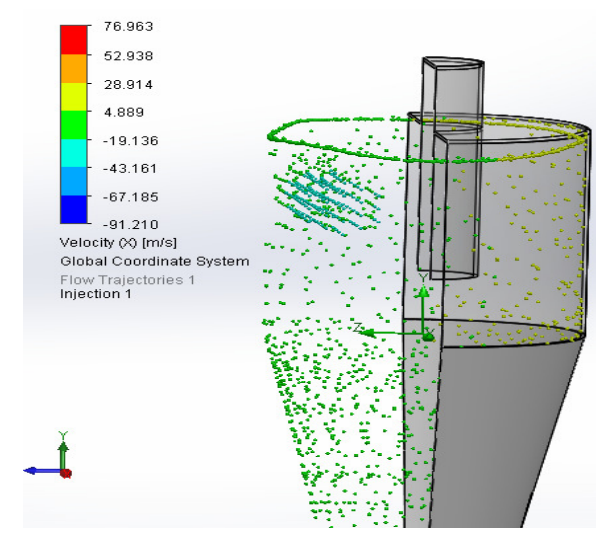

b) Trace of dust particles

Fig. 5. Presentation of trajectories inside cyclone

The following Figures 6-9 show the obtained results of various air characteristics, where the trajectory 6 is a trajectory, which reaches the vortex zone and the trajectory 16 is an air trajectory, which is selected from the outlet side, the values of the characteristics obtained are length-dependent travelling towards the evacuation.

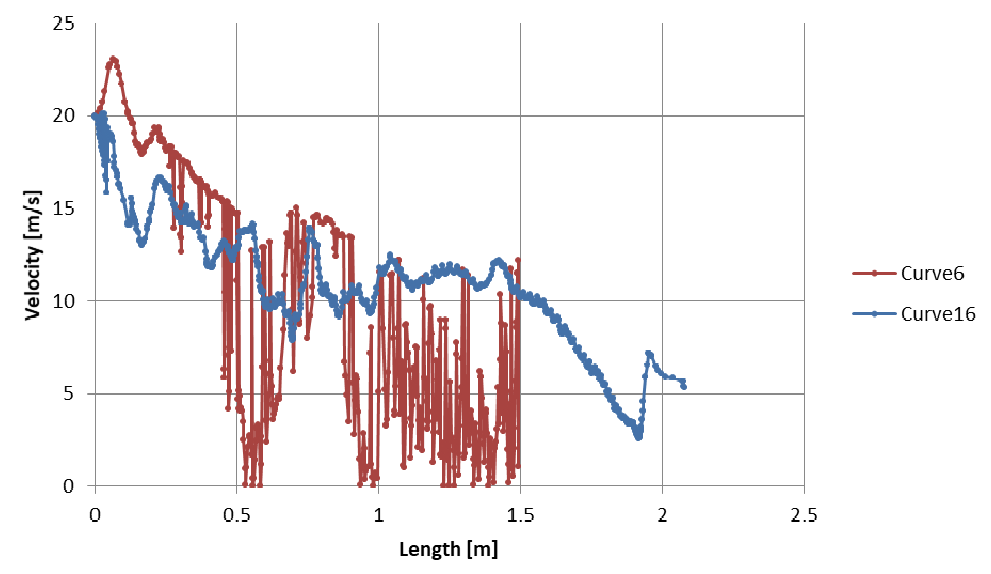

Fig. 6. Velocity variation depending on the distance traveled by path

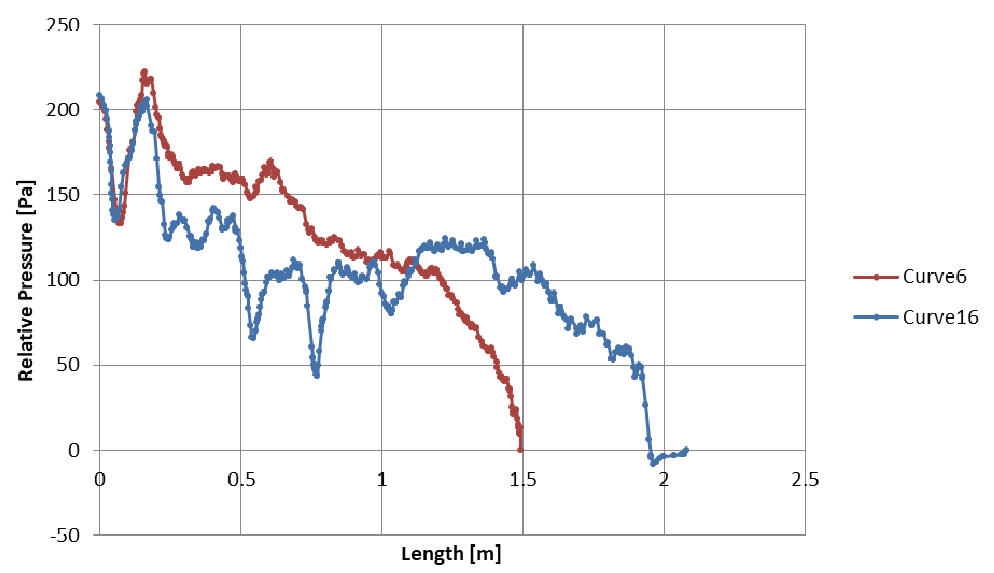

Fig. 7. Relativve pressure variation depending on the distance traveled by path 


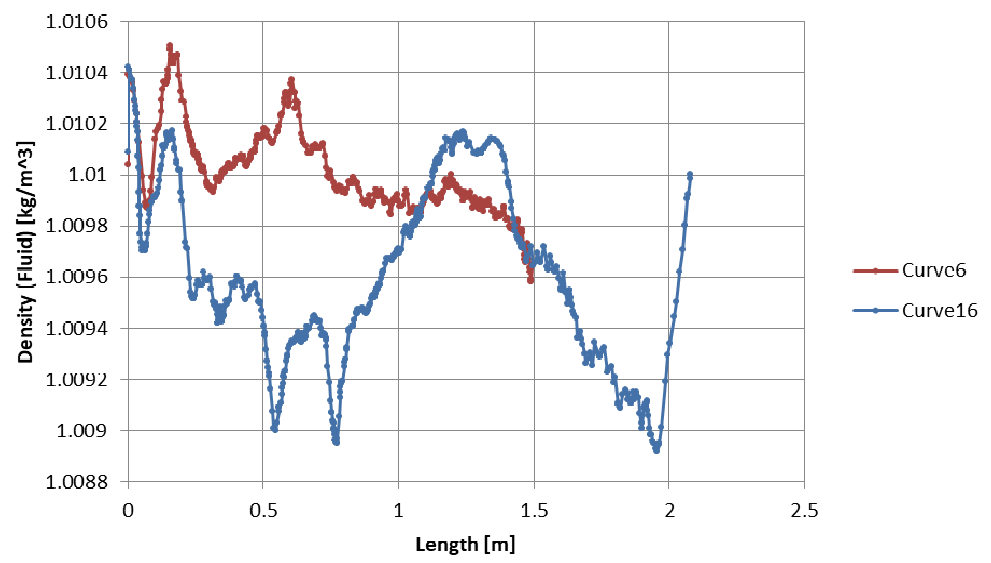

Fig. 8. Density variation depending on the distance traveled by path

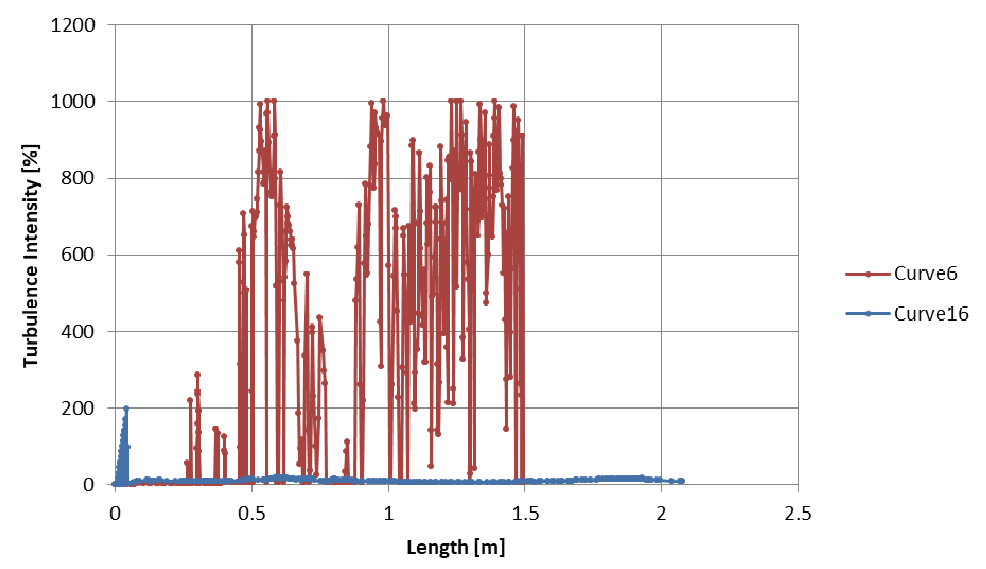

Fig. 9. Turbulence intensity variation depending on the distance traveled by path

\section{Conclusions}

1. The use of state-of-the-art computers and the SCILAB programming environment have allowed to solve a complex calculation of particle motion in the cyclone, obtained by eliminating significant simplifications from the most representative models of calculation from the literature, as well as comparing it with one of the simplest models from the literature. Thus, a dynamic particle study was conducted for dust, with a visual impact on the cyclone's operation. This study leads to a more accurate understanding of the complex phenomena that occur in the dust separation process in the cyclone and determining more accurately the factors and conditions that increase the efficiency of the cyclone, thus contributing to energy efficiency of the dusting facilities.

2. The scientific originality is that of observing using this type of simulation, if dust particles exit through the air outlet and not through the dust outlet (in this simulation we can see in the upper side of Figure 5.b that it does not happen in the case of our cyclone), and with this type of simulation you can measure, as in Figure 6, various characteristics inside the cyclone, for example, if we have excessive increase of these characteristics, we will optimize the constructive parameters of the cyclone.

\section{Acknowledgements}

This work was funded by the Ministry of Research and Innovation, within the project entitled "PN 18 30.01.02: "Eco-innovative system with the "deflector" for the establishment the crops", ctr. $18 \mathrm{~N} / 16.03 .2018$.

\section{References}

[1] Blengini G.A., Busto M., The life cycle of rice: LCA of alternative agri-food chain management systems in Vercelli (Italy). Journal of Environmental Management 90, 2009, pp. 1512-1522. 
[2] Snyder C.S., Bruulsema T.W., Jensen T.L., Fixen P.E. Review of greenhouse gas emission from crop production system systems and fertilizer management affects. Agriculture, Ecosystems \& Environment 133, 2009, pp.247-266.

[3] Safa M., Samarasinghe S. CO2 emissions from farm input "case study of wheat production in Caterbury, New Zeland". Environmental Pollution 171, 2012, pp.126-132.

[4] Lichiheb N., Personne E., Bedos C., Barriuso E. Adaptation of a resistive model to pesticide volatilization from plants at the field scale: comparison with a dateset. Atmos. Environ, 2014, pp. 260-268.

[5] Elbert A., Haas M., Springer B., Thielert W., Nauen R. Applied aspects of neonicotinoid uses in crop protection. Pest management Science 64(11), 2008, pp. 1099-1105.

[6] Nuyttens D., Devarrewaere W., Verboven P., Foquè, D. Pesticide-laden dust emission and drift from treated seeds during seed drilling: a review. Pesticide management Science 69, 2013, pp. 564-575.

[7] Altmann R. Poncho: a new insecticidal seed treatment for the control of the major maize pests in Europe. Pflanzenschutz-Nachrichten Bayer (English edition) 56, 2003, pp.102-110.

[8] Greatti M., Sabatini A.G., Barbatini R., Rossi S., Stravisi A. Risk of environmental contamination by the active ingredient imidacloprid used for corn seed dressing. Preliminary results. Bulletin of Insectology 56, 2003, pp. 69-72.

[9] Greatti M., Barbatini R., Stravisi A., Sabatini A.G., Rossi S. Presence of the a.i. imidacloprid on vegetation near corn fields sown with Gaucho dressed seeds. Bulletin of Insectology 59, 2006, pp. 99-103.

[10] Schnier H.F., Wenig G., Laubert F., Volker S., Schmuck R. Hey bee safety of imidacloprid corn seed treatment. Bulletin of insectology 56(1), 2003, pp. 73-75.

[11] Balsari P., Manzone M., Marucco P., Tamagnone M., Evaluation of seeds dressing dust dispersion from maize sowing machines. Crop Protection 51, 2013, pp. 19-23.

[12] Girolami V., Mazzon L., Squartini A., Mori N., Marzaro M., Di Bernardo A., Greatti M., Giorio C., Tapparo A. Translocation of neonicotinoid Insecticides From Coated 16 Seeds to Seedling Guttation Drop: A Novel Way Intoxication for Bees. Journal Econ. Entomol. 102, 2009, pp. 1808-1815.

[13] Giffard H., Dupont T. A methodology to assess the impact on bees of dust from coated seeds. Julius-K" uhn-Arch 423, 2009, pp. 73-75.

[14]Herbst A., Rautmann D., Osteroth H.J., Wehmann H.J., Ganzelmeier H.,. Drift of seed dressing chemicals during the sowing of maize. Aspects of Applied Biology 99 International Advances in Pesticide Application, 2010

[15]Cold Iolanda, Teodosiu Cătălin, Air Purification and Pneumatic Installations, Design Guide, Technical University of Civil Engineering Bucharest, 1997. 